\title{
L'hommage aux défunts.cn. La semaine de la Toussaint chinoise à Shanghai sur Weibo
}

Tribute to the departed.cn. The week of the Chinese All Souls festival on Weibo

Virginie Julliard

\section{CpenEdition}

Journals

Édition électronique

URL : http://journals.openedition.org/edc/6521

DOI : 10.4000/edc.6521

ISSN : 2101-0366

Éditeur

Université Lille-3

Édition imprimée

Date de publication : 1 juin 2016

Pagination : 131-146

ISBN : 978-2-917562-15-4

ISSN : $1270-6841$

Référence électronique

Virginie Julliard, «L'hommage aux défunts.cn. La semaine de la Toussaint chinoise à Shanghai sur Weibo ", Études de communication [En ligne], 46 | 2016, mis en ligne le 01 juin 2018, consulté le 30 avril 2019. URL : http://journals.openedition.org/edc/6521 ; DOI : 10.4000/edc.6521 


\section{L'hommage aux défunts.cn La semaine de la Toussaint chinoise à Shanghai sur Weibo'}

Tribute to the departed.cn The week of the Chinese All Souls festival on Weibo

Virginie Julliard

Laboratoire COSTECH, Université de technologie de Compiègne virginie.julliard@utc.fr

1 Cette étude s'inscrit dans une recherche collective sur les enjeux des pratiques mémorielles innovantes du Web en France, aux États-Unis et en Chine. Éternités numériques. Les identités numériques post mortem et les usages mémoriaux innovants du web au prisme du genre, projet financé par l'ANR et coordonné par Fanny Georges. 
En Chine, une «révolution funéraire» entreprise dès les années 1950 met en œuvre le passage de l'inhumation des corps à leur incinération. Depuis les années 1990, des sites mémoriaux ont été déployés sur le Web par les autorités afin de limiter l'importance symbolique de l'espace physique dans le rite funéraire. Les internautes peuvent y rendre hommage aux défunts. Cet article choisit d'investiguer ceux qui se formulent dans la plateforme de micro-blogage Weibo, à l'occasion de la Toussaint chinoise. L'analyse des messages publiés entre le $1^{\text {er }}$ et le 9 avril 2014 révèle des enjeux socio-culturel, relationnel et expressif.

Mots-clés : écriture numérique, hommages en ligne, défunts, Toussaint chinoise, Shanghai, Weibo.
Starting in the 1950s, China implemented a "funeral revolution" by replacing traditional burial with cremation. Since the 1990s, memorial websites have been created by the authorities to limit the symbolic importance of the physical space in the funerary rite. On these websites, users can pay tribute to the deceased. This article investigates messages written and posted on the micro-blogging platform Weibo, on the occasion of the Chinese All Souls festival. Analysis of the messages published between April $1^{\text {st }}$ and April9 $9^{\text {th }}, 2014$ reveals socio-cultural, relational and expressive issues.

Keywords: digital writing, online tributes, departed, Chinese All Souls, Shanghai, Weibo. 
× Il fait noir grand-mère, on vient te voir,

Message posté sur Weibo la veille de la Toussaint chinoise.

La communication avec les défunts prend différentes formes: dialogues silencieux, hommage à voix haute, en particulier dans les cimetières (Dow et al., 2005), ou encore lettres manuscrites et messages vocaux sur le répondeur téléphonique du défunt (Despret, 2015). À partir des années1990, l'hommage aux défunts se numérise avec les premiers cyber-cimetières. L'usage du Web pour communiquer avec l'au-delà se développe durant la décennie suivante (Georges, 2013), dans un contexte d'émergence d'applications relationnelles sur le Web, et plus particulièrement de pratiques techno-spirituelles pour la conduite de pratiques religieuses (par ex. Douyère, 2011; Jonveaux, 2013), notamment dans un contexte de deuil (Bell, 2006; Duteil-Ogata et al., 2015). Parallèlement, les internautes se saisissent du Web participatif (par ex. YouTube) pour y ériger les premiers "sanctuaires numériques spontanés» (Paton et Figeac, 2013). Ces phénomènes s'accélèrent depuis les années 2010, les profils à visée mémorielle créés post mortem et les profils persistant après le décès des internautes dans des réseaux sociaux numériques (RSN) tels que Facebook, ou encore l'apparition de services numériques innovants (outils pour constituer soi-même sa mémoire numérique, services des derniers messages) (Georges et Julliard, 2014a) offrant de nouveaux lieux de mémoire et de communication entre les vivants et les morts. On y observe de nouvelles formes d'éloge post mortem, généralement écrites et persistantes. La dynamique d'innovation dans le champ des usages du Web n'est pas sans lien avec la montée contemporaine de l'individualisme (Ehrenberg, 1998). Les notions d'«individualisme expressif » (Allard \& Vandenberghe, 2003) et d'«individualisme connecté» (Flichy, 2004), mettent ainsi en lumière le double mouvement en jeu dans les applications relationnelles sur le Web: I'intérêt commercial des producteurs d'applications rencontrant le désir des usagers de façonner leur identité et d'être reconnus. Avec le Web, et qui plus est le Web participatif, on assisterait donc à un phénomène de subjectivation croissante de l'hommage aux défunts. Cette hypothèse sera travaillée sur le terrain chinois, où la réforme funéraire qui entreprend de remplacer l'inhumation des corps par leur incinération s'accompagne de nouvelles formes de rituels ${ }^{2}$ en ligne (Kong, 2012) encouragées par le gouvernement. L'hommage (en ligne) est ici entendu au sens large, englobant une variété de pratiques d'écriture ${ }^{3}$ autour des défunts depuis la remémoration de moments partagés, jusqu'aux prières en passant par l'expression de sentiments. Les hommages peuvent être compris comme autant d'interprétations

2 Je distingue le rite, comme pratique vécue (ce que les gens «performent»), du rituel, comme codification normative d'un type de pratique sociale (ce qui est défini formellement) (Grimes, 1990).

3 Mon acception d'«écriture» intègre les textes, les images, les vidéos, les signes écrits utilisés comme symboles graphiques, ainsi que les effets typographiques (mise en capitales, ponctuation, ASCII art). 
de ce qu'il convient d'écrire au mort ou à propos du mort dans un dispositif d'écriture numérique, résultant d'un jeu subtil avec les normes imposées par la tradition, la réforme funéraire et les arrangements des individus, aussi bien que d'une appropriation du site Web par les internautes (Jeanneret, 2006) qui peut trahir un désir d'expression de soi (Allard \& Vandenberghe, 2003). Quels enjeux révèlent les hommages aux défunts sur le Web chinois et observe-ton des phénomènes de subjectivation à l'occasion de ces hommages? Pour répondre à ces questions, j'ai analysé un corpus de messages publiés durant la semaine de la Toussaint chinoise (Qingmíng), moment particulièrement propice à la vénération des ancêtres, sur le RSN le plus important de Chine: Sina $W_{e i b o}{ }^{4}$. Après avoir exposé brièvement le contexte de l'étude, je reviendrai sur les difficultés méthodologiques que j'ai rencontrées, puis je présenterai les trois enjeux que l'étude des hommages en ligne révèle.

\section{1. \\ Contexte: "révolution funéraire» et pratiques numériques en Chine}

Depuis 1949, le gouvernement chinois a engagé une réforme funéraire qui vise la cérémonie funèbre, les règles d'héritage et le culte des ancêtres, afin de lutter contre les «superstitions féodales » et de dissoudre l'organisation lignagière et la hiérarchie sociale. En1956, une circulaire impose le passage de I'inhumation des corps à la crémation, avec inhumation d'une urne cinéraire ou dispersion des cendres, rompant ainsi avec des rituels funéraires millénaires qui s'appuient sur le maintien de l'intégrité du corps du défunt. Ces réformes ont été mises en œuvre par le biais de méthodes coercitives ${ }^{5}$, en particulier dans les grandes villes, où il est plus aisé de contraindre les familles à respecter les normes modernes (Goossaert et Fang, 2008). Pour accompagner ces réformes et limiter l'importance symbolique de l'espace physique dans le rite funérairel'objectif visé étant de faire accepter, à terme, la dispersion des cendres-le gouvernement a mis en ligne des mémoriaux ${ }^{6}$, dans lesquels les internautes peuvent créer un espace de mémoire, fleurir une tombe virtuelle, brûler de

4 Créée en 2009 par Sina.com, le $3^{\text {e }}$ plus gros opérateur de sites Web de Chine (Sautedé, 2010).

5 Durant la révolution culturelle, les cimetières de Shanghai sont saccagés et des crémations forcées et collectives sont organisées (Bellocq, 2013). Plus récemment, la mise en œuvre brutale de la réforme funéraire dans certains cantons ruraux a conduit plusieurs personnes âgées à se suicider, soit après que la police a détruit les cercueils qu'ils avaient mis plusieurs années à acheter, ruinant tout espoir d'inhumation des corps complets (illégale mais parfois réalisée avec la complicité des autorités locales), soit avant la mise en application de la réforme dans leur village. L'affaire a suscité une vive émotion sur le Web en 2014 (entretien, anthropologue, 29/10/14).

6 Par ex., Earth village: www.ev991.com 
l'encens, déposer des bougies et rédiger des hommages. De tels mémoriaux sont également proposés par des entreprises de pompes funèbres qui les enrichissent de nouvelles fonctionnalités: les proches peuvent ainsi reproduire le geste rituel de balayer les tombes, par exemple, ce geste renvoyant à celui réalisé le jour de Qingmíng. La célébration de cette fête, fixée au premier weekend d'avril selon le calendrier lunaire, est revitalisée depuis 2008, lorsque le gouvernement déclare un jour férié pour cette occasion, portant le week-end à trois jours. Les rites traditionnels sont modernisés pour correspondre à la sobriété désormais prônée en matière funéraire: les objets que l'on peut brûler en offrande sont réglementés, le dépôt de fleurs, inexistant au préalable, est encouragé (Bellocq, 2013). Les cyber-cimetières accompagnent ce mouvement de «retour» vers une tradition chinoise expurgée des "superstitions". Si le recul des espaces consacrés à la mort s'accompagne d'une inflation des espaces qui lui sont consacrés en ligne, ceux-ci ne rencontrent pas, toutefois, le succès escompté (Kong, 2012). J'ai donc voulu m'intéresser aux hommages spontanés qui s'expriment dans les RSN.

En 2013, la Chine comptait 591 millions d'internautes dont $91 \%$ possédant un compte sur un RSN7. Mon choix s'est porté sur Weibo, considéré comme le plus important d'entre eux avec plus de 500 millions d'utilisateurs ${ }^{8}$. Proche de Twitter, il permet de publier des messages courts de 140 caractères, mais il possède des fonctionnalités supplémentaires, telles que la possibilité de personnaliser les pages profils ou de créer de véritable fils de discussion. 50 millions de messages étant publiés par jour sur cette plateforme ${ }^{9}$, il était impossible de recueillir et d'étudier l'ensemble de ceux publiés au cours d'une semaine. J'ai donc décidé de restreindre l'analyse aux seuls messages postés à Shanghai. Le choix de cette ville se justifie à plusieurs titres. Tout d'abord, la politique extrêmement volontariste du gouvernement chinois en matière de funéraire soulève des enjeux sociaux, politiques, éthiques et économiques spécifiques dans la mégalopole la plus peuplée de Chine continentale ${ }^{10}$. À Shanghai, où l'on compte 100000 décès par an (Bellocq, 2013), la réforme funéraire est suivie à la lettre, et la crémation est obligatoire-sauf pour les Chinois d'outremer et les minorités musulmanes - contre une moyenne de $50 \%$ pour le reste du pays (Goossaert et Fang, 2008). De plus, avec $70 \%$ d'habitants connectés, Shanghai a le taux de pénétration d'Internet le plus élevé de Chine

7 Olivier Verot, «20 chiffres sur le numérique en Chine», 12/12/13, http://www. journaldunet.com/ebusiness/expert/56043/20-chiffres-sur-le-numerique-enchine.shtml

8 Willis Wee, «Sina Weibo Passes 500 Million Users, But Needs to Monetize More on Mobile», Techninasia, 20/02/13, https://www.techinasia.com/sina-weibo500-million-users-but-not-monetizing-mobile

9 Verot, op.cit.

10 Shanghai compterait 25 millions d'habitants, mais ce chiffre est à prendre avec précaution car il est difficile de dénombrer les migrants vivant en situation irrégulière. 
et se situe dans la moyenne des métropoles européennes (CNNIC, 2013), ce qui présente un avantage en terme de comparabilité avec le terrain français étudié par ailleurs (par ex. Georges et Julliard, 2014a). Enfin, le réseau des universités de technologie françaises a un partenariat avec l'université de Shanghai (SHU), qui s'est concrétisé par la création de la plateforme de recherche ComplexCity, où j'ai trouvé des partenaires pour mener à bien cette étude ${ }^{11}$.

\section{2. \\ Étudier I'hommage aux défunts sur le Web Chinois: précisions méthodologiques}

244664 messages ont été collectés sur Weibo entre le $1^{\text {er }}$ et le 9 avril2014cette année là, Qingmíng était célébré le dimanche 6 avril et le lundi 7 était férié. Ces messages ont tous été postés à Shanghai par des comptes n'ayant pas désactivé la géolocalisation ${ }^{12}$. Pour mener une analyse des messages la plus fidèle possible en l'absence de sinologue, l'équipe a consacré le premier temps de l'enquête à l'exploration du terrain. J'ai conduit quatre entretiens exploratoires auprès de sociologues et d'anthropologues de SHU spécialistes des pratiques numériques ou funéraires, puis, avec Fanny Georges ${ }^{13}$, nous avons mené vingt entretiens sur le thème de l'hommage en ligne aux défunts auprès d'étudiants de SHU (dix étudiantes et dix étudiants) ayant un compte Weibo, vivant sur le campus ou à proximité, et âgés de 22 à $26 a^{14} s^{14}$ Le choix des personnes interrogées s'est fait aussi bien en raison de la faisabilité des entretiens - réalisés sur le campus et en Anglais - que de la proximité des enquêtés avec le profil des utilisateurs de Weibo, dont $70 \%$ ont moins de $30 \mathrm{ans}^{15}$.

11 Je remercie Monzen Tzen, directeur de I'UTSEUS, Fabien et Laëtitia Pfaënder (ComplexCity), Wan Wanggen (Smartcity Laboratory), et ses étudiants qui ont collecté les messages, Sun Xiulin (School of Sociology and Political Sciences) et son étudiante Zhang Can qui a recruté les personnes interrogées et m'a assistée lors des visites sur le terrain et des entretiens.

12 Les ingénieurs de ComplexCity ont opté pour une collecte des messages en temps réel via l'API (interface de programmation applicative, en Français) Weibo. Celle-ci imposant une limite de 1000 requêtes par heure et par machine, ils ont parallélisé les accès à l'API sur trois machines. Cette collecte a ensuite été complétée par une collecte a posteriori. Il est cependant impossible de connaître avec exactitude le pourcentage des messages géo-localisés sur I'ensemble des messages et ce que l'API Weibo renvoie, I'analyse ne prétend donc pas être exhaustive. La méthode de captation est détaillée en ligne: http://complexcity.org/studies/2014-05-26-weibo-spatial-and-temporal-data. html

13 Qu'il me soit ici permis de la remercier.

14 Notés E1, E2, etc. dans l'article. L'analyse de ces premiers entretiens m'a convaincue d'en réaliser 20 supplémentaires auprès d'un public plus âgé, et donc plus susceptible d'avoir été associé à des rituels funéraires, et en Chinois, afin de réduire le coût d'accès induit par la barrière linguistique. Ces entretiens sont en cours de traitement.

15 Olivier Verot, Op.cit. 
Dans la mesure où la censure sévit sur le Web chinois et où la mort reste une affaire privée, les entretiens m'ont permis d'éclairer plusieurs aspects de l'objet de recherche échappant à l'analyse de corpus (ce qui ne peut pas être publié, ce qui a été supprimé). Celle-ci a débuté à l'issue de la phase d'exploration. La majorité des messages récoltés ne concernant pas l'hommage aux défunts, j'ai filtré la base initiale pour ne retenir que les messages contenant les caractères d'un des 22 mots-clés ou expressions se rapportant à la mort ou à la vénération des ancêtres en Chinois simplifié ${ }^{16}$. L'analyse porte sur les 7801 messages ainsi obtenus. La traduction de ces messages ${ }^{17}$ n'a pas toujours permis de lever leur ambiguïté du fait de la déconnexion d'avec le contexte de publication - j'ai travaillé sur les messages reportés dans une base de données et non sur les messages tels qu'ils apparaissent aux internautes sur leurs navigateurs-, de leur brièveté, mais aussi de l'«opacité» de la langue chinoise et du système d'écriture.

Le Chinois n'est pas une langue flexionnellle, il n'admet donc ni conjugaison ni accord, et les caractères sont invariables (Alleton, 2005). Par ailleurs, le sujet du verbe est parfois implicite. Ainsi, un même message peut être traduit de différentes manières: "aller balayer les tombes" dans une traduction mot à mot, peut se comprendre comme une déclaration à la première personne, mais sans la certitude que confère le contexte d'une discussion en face à face ou d'une production écrite plus longue. De plus, le Chinois possède plusieurs niveaux de lecture: dans le cas du huíwén (sorte de palindrome), par exemple, les caractères d'une phrase peuvent s'écrire dans un ordre différent tout en signifiant la même chose. Qui plus est, les internautes chinois expriment souvent leurs sentiments en vers, ce qui rend la traduction plus incertaine encore, l'écriture chinoise permettant «des jeux de 'mots' très particuliers par un retour de la langue orale sur sa transcription" (Calvet, 1996, 103). Si les caractères simples ne notent pas, en général, des sons mais des sens, certains d'entre eux peuvent jouer un rôle phonétique en composition:

$\times \quad$ Avec le caractère du cheval ma(A), et celui de la femme, j'obtiens ma, la mère $(B), c^{\prime}$ est-à-dire la femme qui se prononce à peu près comme le cheval. Le même cheval combiné avec la bouche, donne ma, une particule interrogative $(C)$. Combiné avec le caractère de la pierre, le cheval me donne ma, poids ou chiffre(D), et combiné avec deux bouches, il donne ma, insulte(E). Dans tous les cas, la prononciation est assez proche, au

16 清明 (Qingming), 墓 et 坟 (tombes), 死 (mourir - peut se combiner avec d'autres caractères pour signifier mort ou défunt ou suicide), 亡 (mort), 遗体 (dépouille), 骨灰 (cendres), 葬 (enterrer-entre dans la composition d'autres mots tels que funérailles), 火化 (crémation), 自杀 et 寻短见 (se suicider), 自尽 et 自 殘 (suicide), 逝者 (défunt), 自缢 (se pendre), 结束生命 (fin de vie), 悼念 (pleurer quelqu'un), 祭祖 (culte des ancêtres), 默哀 (hommage silencieux), 永远离开 (disparu à jamais), 沉痛哀悼 (deuil), 节哀顺变 (retenez votre chagrin, acceptez le destin).

17 Réalisée par Jiayu Yan, étudiante en master et stagiaire du projet. 
ton près, et le caractère initial sert de phonétique, tandis que l'autre indique le sens (ibid., 95).

Les internautes jouent avec cette homophonie pour contourner la censure, remplaçant certains caractères par d'autres se prononçant de manière similaire (cf. Han Han, 2012). Enfin, en raison de la dimension graphique des caractères, "la poésie se dessine, et se regarde autant qu'elle se lit» (Calvet, 1996, 101). Cela ajoute à la difficulté de rendre l'ensemble des informations contenues dans un message qui prendrait la forme d'un poème. Toutefois, lorsque les internautes empruntent leurs vers à des poèmes connus, il est possible de confronter les traductions existantes pour se faire une idée plus juste de la manière dont ils peuvent être interprétés. Ces précisions faites, abordons les enjeux que révèlent les nouvelles formes d'hommage aux défunts.

\section{3. \\ Les enjeux socio-culturels révélés par les nouvelles formes d'hommage}

Les rituels traditionnels d'hommage aux défunts sont circonscrits dans l'espace (la sépulture, l'autel des ancêtres ${ }^{18}$ ) et le temps (funérailles, Toussaint, anniversaires de décès). Toutefois, comme je l'ai précisé plus haut, le gouvernement entend limiter l'importance symbolique de l'espace physique dans le rite funéraire. Par ailleurs, un grand nombre de Chinois est dans l'incapacité de se rendre sur les lieux de vénération traditionnels lors de Qingmíng. Les raisons que les internautes invoquent pour expliquer leurs difficultés à rentrer chez eux sont éclairantes du point de vue des mutations sociales et culturelles que connaît la Chine contemporaine. En premier lieu, l'insuffisance des infrastructures et la pression démographique rendent les déplacements particulièrement problématiques durant les fêtes nationales. Dans les mégalopoles, les transports en commun sont pris d'assaut, et les embouteillages sont très impressionnants durant le week-end de Qingmíng, plusieurs internautes s'en plaignent sur Weibo. En deuxième lieu, la dispersion des familles sur l'immense territoire chinois rend difficile leur réunion à l'occasion d'un week-end de trois jours. Seuls deux étudiants interrogés ont pu célébrer Qingmíng en famille. Ils comptent parmi les dix dont les parents vivent à Shanghai ou à proximité. En troisième lieu, de nombreux internautes indiquent qu'ils doivent travailler le week-end de Qingmíng et ne peuvent prendre du repos. Deux étudiants interrogés ont également avancé cette explication. En quatrième lieu, certains internautes indiquent qu'ils n'ont tout simplement pas les moyens d'entreprendre

18 Les Chinois considèrent qu'à la mort du défunt, la composante yang de l'âme quitte le corps pour rejoindre la tablette disposée sur l'autel des ancêtres, tandis que sa composante yin reste dans la dépouille. 
le voyage. Cette raison n'a été avancée par aucun étudiant interrogé.

Cette incapacité à se déplacer conduit les Chinois à «négocier» avec les règles qui encadrent la célébration de cette fête ${ }^{19}$. Par exemple, un service de petites annonces dédié au recrutement de personnes pour l'entretien des sépultures a récemment vu le jour sur le site Taobao, lequel service contrevient à la règle selon laquelle les tombes doivent être balayées par quelqu'un de la famille des défunts. Par ailleurs, de nouvelles formes d'hommage voient le jour pour pallier l'hommage traditionnel «en présence» (sous-entendu en présence d'un aspect de l'âme du défunt) qui doit être accompli le jour de Qingmíng. Il peut s'agir de vénérer ses ancêtres à un autre moment (le week-end précédent ou durant les vacances du Nouvel an chinois) ou en un autre lieu que les cimetières (durant Qingmíng, mais dans sa ville d'habitation), comme en témoignent les messages et les entretiens. Weibo constitue un des nouveaux lieux d'hommage aux défunts et de communication entre les vivants et les morts qui ne sont pas contraints par les conditions de temps et d'espace définis par les rituels classiques dans des lieux déterminés:

× J'espère que mes grands-parents vont bien, au paradis. Je ne peux pas rentrer, brûler de l'encens et du papier monnaie pour vous ce Qingmíng, mais je prie toujours pour vous et je pense à vous (message, 04/04/14).

Les entretiens le confirment:

$\times \quad$ C'est pratique de pouvoir rendre hommage aux défunts en ligne, pour montrer notre respect. Ce n'est pas si facile de rentrer dans notre ville d'origine [...] de respecter les cérémonies traditionnelles (E12, étudiante, 25 ans, 23/04/14).

Sur Weibo, l'hommage aux défunts peut prendre la forme d'épitaphes, de nombreux messages comportant l'expression "Repose en paix», ou de prières associés à des hommages silencieux (icônes représentant des mains jointes, des bougies ou des fleurs, idéogrammes signifiant «bougie», "cœur » ou «fleur» insérés dans des crochets comme pour évoquer l'icône correspondante). Par ailleurs, I'hommage en ligne peut s'articuler avec d'autres formes d'hommage ou de communication entre les vivants et les morts (la prière ou le dialogue intérieur, cf.le message ci-dessus). Le message en épigraphe de cet article, dans lequel une internaute annonce à son ancêtre qu'elle vient se recueillir sur sa tombe, témoigne également que la communication entre les vivants et les morts sur le Web peut redoubler I'hommage traditionnel «en présence».

Dans le contexte d'expansion de la société de consommation, certains internautes et étudiants chinois expriment aussi leur souhait de profiter

19 Ces règles sont rappelées dans plusieurs articles publiés début avril «Je suis en train de lire 'Huit choses interdites pour balayer les tombes', Lisez-le!» (message, 03/04/14). L'article cité est consultable en ligne:

http://t.cn/8sJn3FX 
pleinement de ce week-end prolongé pour s'adonner à des pratiques de loisirs. De fait, nombreux sont les internautes qui profitent de Qingming pour faire du tourisme. Plus de 610000 messages postés sur Weibo (53 dans la base) comportent le hashtag \#oùalleràQingmíng\#, lequel indexe des contributions évoquant des lieux où les internautes se sont rendus pour l'occasion. Mais ici encore, les pratiques s'hybrident, car plusieurs de ces contributions évoquent le mémorial du massacre de Nankin, une des destinations de prédilection des Shanghaïens durant Qingmíng, qui célèbre les victimes de l'occupation japonaise. Le voyage touristique s'apparente alors à une forme de pèlerinage à l'occasion duquel les Chinois vont rendre hommage aux martyrs de la Nation. II semble que l'âge soit un facteur explicatif du désintérêt pour la célébration de Qingmíng. Plusieurs étudiants interrogés ont expliqué que c'étaient à leurs parents d'honorer leurs grands parents, car c'est aux enfants des défunts qu'il revient de conduire le rituel. D'autres étudiants estiment que l'hommage aux défunts est une forme de superstition, radicalisant le discours du gouvernement. Toutefois, ce désintérêt peut être transitoire, tant que l'on a pas soi-même expérimenté la mort d'un proche ou la vieillesse: "Auparavant je pensais que ma mère était superstitieuse de balayer les tombes et que je ne ferai pas ça quand je serai grand, mais quand j'entends à la radio qu'on discute sur les balayeurs de tombeaux rétribués, je pleure. Je suis vieux et je commence à comprendre des choses que je ne comprenais pas avant» (message, 02/04/14).

\section{4. L'enjeu relationnel de l'hommage aux défunts en ligne}

Dans un contexte de forte diaspora, le Web offre une multiplicité de services permettant de garder contact avec les membres de sa famille restés dans la province d'origine ou partis s'établir dans une autre province, voire à l'étranger. We Chat, une application mobile permettant d'échanger de manière privée avec des fonctionnalités similaires à celles de Facebook («mur», partage de photos et de contenus), semble particulièrement adapté aux échanges familiaux et amicaux, comme en témoignent tous les étudiants interrogés. Weibo, RSN plus "ouvert», sert également, quoique dans une moindre mesure, à maintenir les liens avec ses proches. L'entretien des liens avec la famille est tellement important qu'il autorise quelques entorses au rituel observé durant Qingmíng. Une étudiante interrogée nous a ainsi rapporté les discussions suscitées par la publication de photographies représentant des tombes sur We Chat:

× Cette année, pour Qingmíng, une amie a posté la photo d'une tombe et a reçu de nombreux commentaires disant «Tu ne devrais pas faire ça, c'est une agression, ce n'est pas approprié de publier ce type d'image» [...]. Elle a répondu qu'elle avait posté cette photo pour que son frère et sa sœur, qui vivent en Amérique, puissent voir la tombe de leurs grands- 
parents [...]. Du coup, les autres ont dit qu'ils comprenaient (E19, étudiante, 24 ans, 25/04/14).

Traditionnellement, la mort est une affaire familiale et privée, et il est plutôt inapproprié d'évoquer le décès d'un proche et de lui rendre un hommage public, a fortiori sur Weibo qui instrumente la publication et la persistance des productions, et dont témoigne la majorité des entretiens:

$\times$ Rendre hommage en ligne est approprié pour les personnages publics[...], cela a un sens dans le cas d'accidents ou de tremblements de terre, mais pas pour ma famille et mes amis, ça, c'est privé (E10, étudiante, 24 ans, 23/04/14).

Par ailleurs, nombreux sont les Chinois qui refusent de parler des morts parce qu'ils considèrent que le sujet est trop triste ou que cela va leur porter malheur. Bien que la commémoration en ligne de personnalités et de victimes militaires et civiles, de guerre ou d'accidents, soit admise, voire encouragée par les autorités, elle est souvent mal vécue par les familles des défunts (E7, étudiante, 25 ans, 23/04/14), celles-ci pouvant demander la suppression d'un hommage en ligne à son auteur (entretien, sociologue, 17/06/14). Cependant, tous les étudiants ne partagent pas ce point de vue: "Pour moi, je pense que c'est une marque de tendresse que de rendre hommage à ses proches en ligne» (E19). Ici encore, l'âge semble être un facteur susceptible d'influencer l'interprétation plus ou moins stricte des règles en matière d'hommage. C'est du moins une éventualité avancée par plusieurs des étudiants interrogés. "Je pense que la génération de mes parents considère que ce n'est convenable de rendre hommage à nos proches en ligne, mais pour les gens de ma génération, c'est $\mathrm{OK} »(\mathrm{E} 12)$.

Si l'hommage en ligne permet d'entretenir les liens entre les vivants, il permet aussi d'assurer la persistance des liens entre les vivants et les morts. En Chine, il s'inscrit dans la continuité de l'hommage traditionnel aux défunts, lequel constitue un système de don et contre-don (en retour de l'attention que ses descendants lui portent, l'ancêtre sert leurs intérêts d'outre tombe) (Javary, 2000). Le nettoyage des tombes et les offrandes aux ancêtres s'accompagnent de requêtes qui concernent essentiellement la santé, le bonheur familial et la réussite sociale. Sur Weibo, les internautes s'adressent souvent à leurs morts pour leur demander ou les remercier de les protéger: "Grands-parents, je vous remercie de nous protéger du ciel» (message, 04/04/14). Leurs requêtes se doublent parfois d'excuses parce que les internautes n'ont pas rendu hommage à leurs défunts de manière conforme: "Cela fait plusieurs années que je n'ai pas balayé la tombe de mon grand-père pendant la fête de Qingmíng, pardonnez moi, grand-père, j'espère que vous nous protégez dans le ciel» (message, 03/04/14). Pour certains, les hommages en ligne étant plus spontanés, ils seraient plus désintéressés (E12). Il arrive ainsi que les hommages en ligne prennent un tour plus personnel, les internautes se saisissant de Weibo pour exprimer des regrets de ne pas avoir rendu une dernière visite ou de ne pas 
avoir su manifester leurs sentiments:

$\times \quad$ J'ai toujours des regrets quand je pense à vous, maintenant que je ne peux plus vous accompagner, j'ai manqué de vous voir la dernière fois. Un an plus tard, ma grand-mère, vous devez être très heureuse dans le ciel, je pense à vous! (message, 03/04/14).

$\times \quad$ Pardonnez moi, je n'ai pas pu exprimer la douleur de vous perdre, mais je me souviens toujours de votre sourire. S'il était encore temps, je vous embrasserais! Je pense à vous, grand père (message, 04/04/14).

Les internautes s'adressent à leurs morts devant un tiers silencieux - les propriétaires des comptes «amis» ne répondent presque jamais à ces messages qui ne leur sont pas destinés. Toutefois, en performant publiquement de tels hommages, les internautes s'engagent dans un rôle, affirmant un lien particulier avec leurs proches défunts. II est ainsi possible de formuler l'hypothèse que ces hommages leur permettent de s'affranchir des règles particulièrement contraignantes déterminant quels descendants ont un rôle à jouer dans le culte des ancêtres (Captdeville-Zeng, 2009).

\section{5. \\ L'enjeu «expressiviste» de l'hommage aux défunts en ligne}

Sophie Arsène a montré que les internautes chinois se saisissaient du Web participatif pour soulager leur mal-être, dans un contexte de montée de I'individualisme qui voit aussi naître un véritable engouement pour la psychologie (2011). Si certains estiment que la peine ressentie au décès d'un membre de sa famille est un sentiment trop intime pour faire l'objet d'une publication et préfèrent s'adresser au petit groupe des très proches sur We Chat ou se confier à leur journal intime (E15, étudiant, 22 ans, 24/04/14), j'ai pourtant relevé un grand nombre de messages dans lesquels les internautes expriment sans détour leurs sentiments à ce propos. L'hommage en ligne aux défunts est aussi l'occasion de réfléchir sur soi et sur les transformations qui s'opèrent sur soi-même à l'occasion de la perte d'un proche. Sur Weibo l'expression de la peine prend souvent la forme d'un poème inédit ou classique (en particulier la première strophe du poème "Qingmíng » ${ }^{20}$ : «le jour de Qingmíng, une pluie fine tombe, comme des larmes» est citée à 54 reprises dans le corpus). Les internautes expriment également leur amour pour leur famille («ma famille adorée», 01/04/14; "Je commémore mon père, mes grands-parents dans mon cœur. Je les aime et je pense à eux», 05/04/14), voire même pour une célébrité. Ainsi, le $1^{\text {er }}$ avril, jour du $11^{\mathrm{e}}$ anniversaire du suicide de Leslie Cheung, premier 
acteur Chinois à avoir accepté des rôles de personnages homosexuels avant d'apparaître publiquement avec son compagnon, les internautes sont nombreux à lui rendre hommage. Ils lui déclarent leur amour, prient pour qu'il connaisse enfin la paix et retrouve son compagnon dans l'au-delà, et récitent les paroles de chansons romantiques qu'il a interprétées. Très important, le phénomène a donné lieu à des formes d'hommages collectifs que balisent des hashtags, tels que «\#pourcommémorerleonzièmeanniversairedelamortde-Leslie\#», indexant des fils comptant quelques centaines de milliers de messages - d'ailleurs, plusieurs étudiants ont spontanément cité ce cas, comme exemple d'hommage en ligne à une personnalité défunte. La nostalgie d'un temps où l'être cher était encore vivant prend la forme de récits retraçant des souvenirs d'enfance, le sentiment de solitude n'en apparaît que plus vivement:

× J'ai vu un homme âgé regarder attentivement les boissons dans le supermarché, et il en a acheté beaucoup. Je pense que ses petits-enfants rentrent pour la fête de Qingmíng. À la caisse, il était souriant. Je me rappelle de ma grand-mère, il y a longtemps, qui sortait du café très vieux, déjà périmé, et me disait de venir le boire. Il n'y a plus personne qui m'aime autant qu'eux, je pense à eux (message, 02/04/14).

Les internautes expriment également leur peur de mourir ("J'ai pensé tout à coup à la mort et j'ai eu peur, même si je sais que je peux mourir à 80 ans ou 90 ans , message, 06/04/14). Ils la conjurent en s'enjoignant de profiter de la vie: «Épitaphe pour moi-même: vis bien, dépasse-toi. L'éphémère est éternel, je sais que je vais mourir un jour» (message, 05/04/14); "Après avoir balayé les tombes, j'ai fait un tour dans le cimetière et j'ai vu des visages très jeunes ${ }^{21}$. Je dois bien profiter de la vie» (message, 07/04/14). La publicisation de ces résolutions en augmente la portée, dans la mesure où les internautes s'engagent devant la communauté de leurs "amis». Ce besoin d'exprimer ses sentiments, quels qu'ils soient, de se raconter pour se produire comme sujet et d'être reconnu par autrui vient aussi recomposer les frontières des espaces public et privé en matière de mort et d'hommage aux défunts, puisqu'il encourage les internautes à rendre publics des faits et des sentiments qui, traditionnellement, par respect ou par superstition, ne doivent pas l'être.

\section{6. \\ Conclusion}

L'analyse des messages postés sur Weibo durant la première semaine d'avril2014 à Shanghai a mis au jour certains des enjeux socio-culturel, relationnel et expressiviste de l'hommage aux défunts en ligne. En particulier, j'ai montré

21 En Chine, il est fréquent que la photographie des défunts figure sur la pierre tombale. 
que des phénomènes de subjectivation étaient observables dans les nouvelles formes d'hommage qui pallient, prolongent, renouvellent et/ou s'articulent avec les formes traditionnelles. II reste qu'il me faut étudier plus avant ces formes dans la perspective d'une comparaison avec le terrain français. Par ailleurs, lors de la phase exploratoire, j'ai identifié un compte référençant les profils de défunts sur Weibo: 逝者如斯夫 dead ${ }^{22}$. Depuis 2011, il a dénombré 600 profils, en avisant ses abonnés par le biais de messages s'apparentant à de brèves nécrologies ou reprenant le dernier message posté par le défunt. Un premier repérage de ces profils a permis de relever des publications postérieures au décès. Une prochaine phase de l'étude portera sur ces annonces et sur l'analyse des profils de défunts qui, à l'instar des profils Facebook que nous étudions avec Fanny George (2014b), accueillent des hommages d'un nouveau genre. 
Bibliographie

Allard L. et VandenbergheF. (2003), «Express yourself! Les pages perso entre légitimation techno-politique de l'individualisme expressif et authenticité réflexive peer to peer», Réseaux, n¹17, pp.191-219.

Alleton V. (2005), L'écriture chinoise, Paris, PUF [1970].

ArsèneS. (2011), Internet et politique en Chine, Paris, Khartala.

Bell G. (2006), "No more SMS from Jesus: Ubicomp, religion and techno-spiritual practices", in Dourish $P$. and Friday A. (eds.), UbiComp 2006: Ubiquitous Computing, Springer Berlin Heildelberg, pp.141-158.

Bellocq M. (2013), «De petits arrangements avec les morts: les rites funéraires à Shanghai, entre tradition et modernité ", in AvelineDubach N. (dir.), La place des morts dans les mégalopoles d'Asie orientale, Paris, Les Indes Savantes, pp.111-134.

Calvet L.-J. (2005), Histoire de l'écriture, Paris, Hachette Littératures.

Capdeville-Zeng C. (2009), «Un lignage chinois aujourd'hui en Chine du Sud-Est (enquête)", Terrains \& travaux, $\mathrm{n}^{\circ} 16, \mathrm{pp} .31-53$.

CNNIC (China Internet Network Information Center) (2013), «Analyse des applications du web social des internautes chinois".

En ligne: http://www1.cnnic.cn/ IDR/ReportDownloads/201302/ P020130221391269963814.pdf, date de la dernière visite: 20 juin 2015.

Despret V. (2015), Au bonheur des morts. Récit de ceux qui restent, Paris, La Découverte.

Douyère D. (2011), "La prière assistée par ordinateur », Medium, n²7, pp.140-154.

DowS., Lee J., Oezbek C., Macintyre, Bolter J.D. \& Gandy M. (2005), «Exploring spatial narratives and mixed reality experiences in Oakland Cemetery», Proceedings of the 2005 ACM SIGCHI International Conference on Advances in computer entertainment technology ACE, pp. 51-60.

Duteil-Ogata, Jonveauxl., KuczynskiL. et NizardS. (dir.) 2015, Le religieux sur internet, Paris, L'Harmattan.

Ehrenberg A. (1998), La fatigue d'être soi, Paris, Odile Jacob.

FlichyP. (2004), "L'individualisme connecté entre la technique numérique et la société", Réseaux, n¹24, pp.17-51.

GeorgesF. (2013), «Le spiritisme en ligne. La communication numérique avec l'au-delà ", Les Cahiers du numérique, $\mathrm{n}^{\circ}$ 3-4, pp. 211-240.

GeorgesF. et Julliard V. (2014a), «Aux frontières de l'identité numérique. Éléments pour une typologie des identités numériques post 
mortem », in SalehI., Bouhaï N. et Hachour $\mathrm{H}$. , Les frontières du numérique, Paris, L'Harmattan, coll. «Global, Local», pp.33-48.

GeorgesF. et JulliardV. (2014b), «La construction de l'identité numérique, le cas des pages dédiées aux défunts sur Facebook", $19^{\mathrm{e}}$ Congrès de la SFSIC, Toulon, Université de Toulon, 5 juin.

Goossaert V. et Fang L. (2008), «Les réformes funéraires et la politique religieuse de l'État chinois", Archives de sciences sociales des religions, $\mathrm{n}^{\circ} 144$, pp.51-73.

Grimes R. (1990), Ritual criticism, Columbia, University of South Carolina Press.

Han H. (2012), Blogs de Chine, (trad. Par H. Denès), Paris, Gallimard.

JavaryC. (2000), "Le culte des ancêtres en Chine», in Tardan-Masquelier Y. et Lenoir F.(dir.), L'Encyclopédie des religions, Paris, Bayard Édition [1997], pp.1932-1936.

JeanneretY. (2006). «Faire avec le faire communicationnel: les prétentions de la sémiotique face à I'horizon des pratiques ", Nouveaux Actes Sémiotiques. Actes de colloques Arts du faire: production et expertise. En ligne: http://epublications.unilim.fr/revues/as/3336, date de la dernière visite: 01/01/2016.

JonveauxI. (2013), Dieu en ligne. Expériences et pratiques religieuses sur Internet, Paris, Bayard.
Kong L. (2012), « No place, New Places: Death and its Rituals in Urban Asia », Urban Studies, $n^{\circ} 49(2)$, pp. 415-433.

Paton N. et Figeac J. (2013), «La commémoration des 'mauvais morts' au sein de sanctuaires spontanés numériques", Les Cahiers du numérique, $\mathrm{n}^{\circ}$ 3-4, pp. 241-270.

SautedéÉ. (2011), «Les réseaux sociaux numériques en Chine: une constellation de petits mondes", Hermès, n59, pp.151-158. 